\title{
Antibiotherapy Removal of an Ingested Fish Bone with Inflammation and Mild Abscess: A Case Report
}

\author{
Jueting Wu*, Jiangtao Fu, Kairui Xu, Chuan Shen, Yuyao Zhang and Jueting Wu \\ Department of Otolaryngology Head and Neck Surgery, China
}

*Corresponding author: Jueting Wu, Department of Otolaryngology Head and Neck Surgery, Lishui People's Hospital, The Sixth

Affiliated Hospital of Wenzhou Medical University, Dazhong Street No. 15, Liandu District, Lishui 323000, Zhejiang, China

\begin{tabular}{l}
\hline ARTICLE INFO \\
\hline Received: 业 March 27, 2019 \\
Published: 㓞 April 03, 2019 \\
\hline Citation: Jueting Wu, Jiangtao Fu, \\
Kairui Xu, Chuan Shen, Yuyao Zhang, \\
Jueting Wu. Antibiotherapy Removal of \\
an Ingested Fish Bone with Inflamma- \\
tion and Mild Abscess: A Case Report. \\
Biomed J Sci \& Tech Res 16(4)-2019. \\
BJSTR. MS.ID.002897.
\end{tabular}

Keywords: Fish Bone; Inflammatory; Abscess; Antibiotherapy

\section{ABSTRACT}

Background: Although most of the foreign body, such as a fish bone can be inadvertently dislodged, severious complications range from mild inflammatory changes to abscess formation and viscus perforation can occurs for the delayed visiting time and unaware of the ingestion history. In such cases, surgery treatment is inevitable.

Case Presentation: A 55-year-old male presented with pain on swallowing after accidental fish bone ingestion 3 days before. We first attempted to remove the fish bone under flexible endoscopy but failed because nothing was detected except significant soft tissue edema on the left lateral laryngopharynx. After conducting contrast-enhanced computed tomography, a 19.5-mm-long linear hyperdensity lying in the left lateral laryngopharyngeal space encircled by a low-density area with fluid was demonstrated, suggestive of ingested fish bone with infection and mild abscess. Operation was our preferred treatment as the infection improved. CT was conducted agained to clarify the acurate spot of the fish bone right before the operation after 5 days of antibiotherapy, and we detected completely free of fish bone in original position. The patient recovered without complications and was discharged on the $7^{\text {th }}$ day.

Conclusion: Early medication treatment including antibiotics for the infection caused by fish bone ingestion can help with the fish bone dislodgement.

\section{Introduction}

Accidental ingestion of a foreign body is a common complaint among patients in the emergency department, account for up to $84 \%$ of the which is fish bone [1]. Patients present with pain on swallowing, rest pain, blood-stained saliva, and sense of obstruction. Although most ingested fish bone will pass through the gastrointestinal tract uneventfully, and the above symptom relieves in a couple of days, approximately 10 to $20 \%$ require endoscopic removal and approximately $1 \%$ require surgery [2]. Even more, delayed diagnosis may lead to sever consequences including deep neck infection or abscess, or even fistula. We herein report a case of an ingested fish bone that caused laryngopharyngeal abscess and eliminated by itself after antibiotic therapy.

\section{Case Presentation}

A 55-year-old male was admitted to our hospital because of pain on swallowing that had begun after accidental fish bone ingestion 3 days before admission. On examination, his body temperature was $38.6{ }^{\circ} \mathrm{C}$, heart rate was 94 beats per minute, blood pressure was 126/80 mmHg, respiratory rate was 19 breaths per minute. Severe throat pain and difficulty in swallowing were present. We inserted gastric tube from nose to keep the energy supply. The laboratory data showed white blood count $10600 / \mathrm{mm} 3$, the C-reactive protein level $35.5 \mathrm{mg} / \mathrm{dl}$. A careful oral examination and flexible endoscopy under local pharyngeal anesthesia were performed, nothing was detected except significant soft tissue edema on the left lateral laryngopharynx. Contrast-enhanced computed tomography scan (CT) images of the neck demonstrated a linear hyperdensity with the length of $19.5 \mathrm{~mm}$,lying in the left lateral laryngopharyngeal space, surrounded by a low-density area with fluid, suggestive of ingested fish bone with abscess (Figure 1). Significant edema and mild abscess showed in the left lateral laryngophary, resulting in mild narrowing of the airway. We therefore assumed that the fish 
bone completely resided inside the edema. Only when the edema gradually vanished, the operation to remove the fish bone can be performed. After 5 days of antibiotherapy, we found the edema on the left lateral laryngopharynx was disappeared under the flexible endoscopy. CT was conducted agained to clarify the accurate spot of the fish bone right before the operation when we detected completely free of fish bone in original position (Figure 2). The patient recovered uneventfully and was discharged on the seventh day.

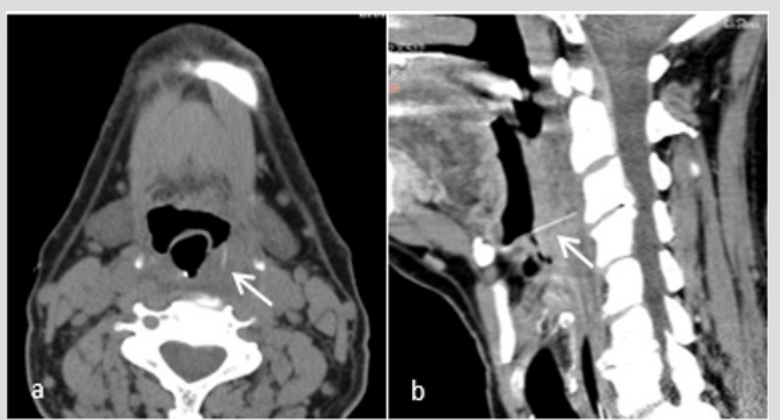

Figure 1: Ingested fish bone on the left hypopharynx. contrast-enhanced CT images of the neck demonstrate a linearhyperdensitylyingin theleftextralaryngopharyngeal space (long arrows) suggestive of ingested fish bone.
a. Axial and
b. Sagittal.

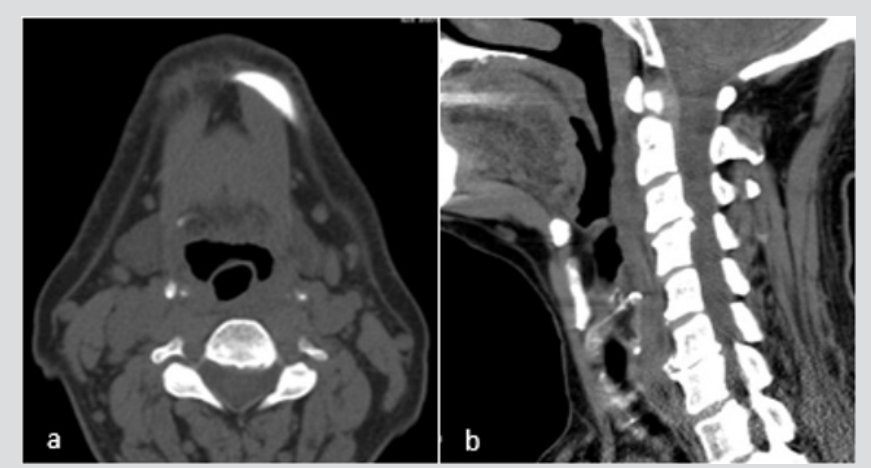

Figure 2: Soft tissue edmema and mild abscess caused by fish bone ingestion vanished and fish bones dislodged.
a. Axial and
b. Sagittal.

\section{Discussion}

Foreign body, especially the fish bone ingestion remains a common clinical problem. About $80 \%-90 \%$ of ingested foreign bodies pass through the gut without discovery within 1 week [3]. Most of fish bones encountered are found above the cricopharyngeal sphincter. A comprehensive physical examination of the oral cavity, oropharynx, and hypopharynx assist by a flexible laryngoscope would detect and remove the fish bone as long as timely treatment. Complications range from mild inflammatory changes to abscess formation, viscus perforation, intestinal obstruction, and bleeding are mostly induced by the delayed visiting time and unaware of the ingestion history. In such cases, surgical intervention is required. CT plays a critical role in diagnosis as it reveals the image of a linear, hyperdense, foreign body corresponding to a bone clearly, let alone its non-invasiveness, early detection of complications and delineation of adjacent structures [4]. Back to our case, instead of seeking clinical attention immediately after the fish bone ingestion, the patient chose to neglect the discomfort and kept a normal diet afterwards, which caused local inflammation and abscess formation. We first attempted to remove the foreign body endoscopically but failed because nothing was detected but the soft tissue edema on the left lateral laryngopharynx.

Contrast-enhanced CT was conducted, a linear hyperdensity with the length of $19.5 \mathrm{~mm}$ at C3 and mild mild abscess formation were demonstrated. After antibiotic therapy for 5 days, pharyngeal symptoms were significantly relieved, both the laryngoscope and CT revealed disappearance of local tissue edema as well as the fish bone. Hence, we suggest an endoscopic examination would be attempted first not only for the diagnosis but also for the removal of the detected foreign body. In the case of fish bone impaction, CT image is important for making a pre-operative plan and an accurate preoperative diagnosis in case there is a high degree of suspicion that the operation is whether indispensable. The treatment consists of endoscopic removal, surgical removal, abscess drainage if necessary, and administration of appropriate antibiotics. Collectively, our case demonstrated that soft tissue edema and mild abscess caused by fish bone ingestion would vanished and fish bones dislodged and passed after treatment with antibiotics. Therefore, early diagnosis and treatment is important, specially medication including antibiotics for the infection which might avoid surgery.

\section{References}

1. Leong HK, Chan RK (1987) Foreign bodies in the upper digestive tract. Singapore Med J 28(2): 162-165.

2. Birk M, Bauerfeind P, Deprez PH, Hafner M, Hartmann D, et al. (2016) Removal of foreign bodies in the upper gastrointestinal tract in adults: European Society of Gastrointestinal Endoscopy (ESGE) Clinical Guideline. Endoscopy 48: 489-496.

3. Kanazawa S, Ishigaki K, Miyake T, Ishida A, Tabuchi A, et al. (2003) A granulomatous liver abscess which developed after a toothpick penetrated the gastrointestinal tract: report of a case. Surg Today 33(4): 312-314.

4. Guelfguat M, Kaplinskiy V, Reddy SH, Di Poce J (2014) Clinical guidelines for imaging and reporting ingested foreign bodies. AJR Am J Roentgenol 203(1): 37-53. 


\section{ISSN: 2574-1241}

DOI: 10.26717/BJSTR.2019.16.002897

Jueting Wu. Biomed J Sci \& Tech Res

(C) (P) This work is licensed under Creative

Submission Link: https://biomedres.us/submit-manuscript.php

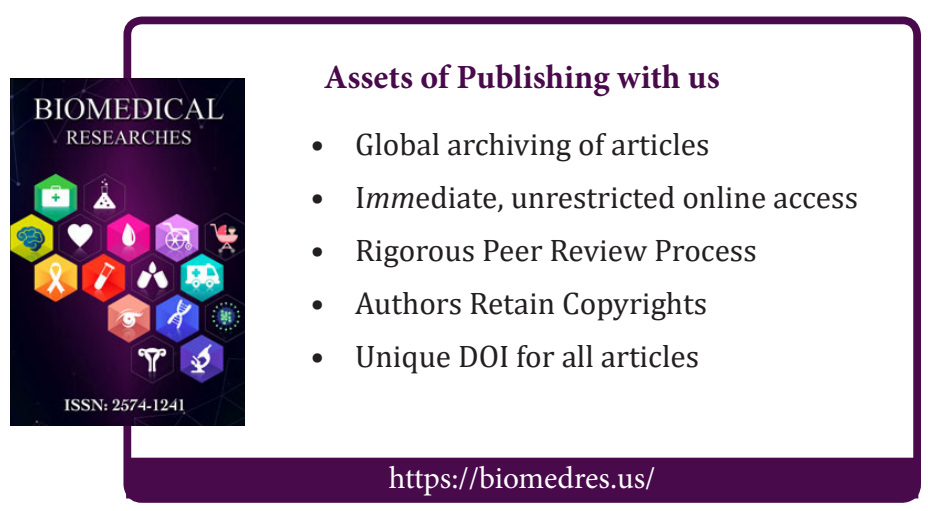

